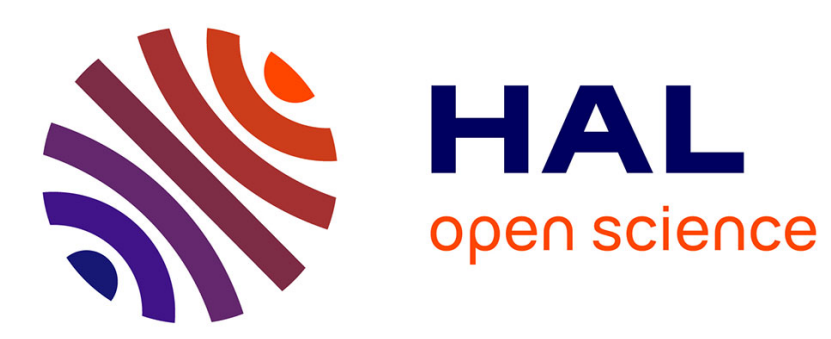

\title{
L'habitat épimagdalénien des Bruyères: essai d'analyse et d'interprétation des structures
}

Bruno Bosselin

\section{To cite this version:}

Bruno Bosselin. L'habitat épimagdalénien des Bruyères: essai d'analyse et d'interprétation des structures. Bulletin de la Société préhistorique française, 1982, 79 (5), pp.138-147. hal-00201592

\section{HAL Id: hal-00201592 \\ https://hal.science/hal-00201592}

Submitted on 14 Jan 2008

HAL is a multi-disciplinary open access archive for the deposit and dissemination of scientific research documents, whether they are published or not. The documents may come from teaching and research institutions in France or abroad, or from public or private research centers.
L'archive ouverte pluridisciplinaire HAL, est destinée au dépôt et à la diffusion de documents scientifiques de niveau recherche, publiés ou non, émanant des établissements d'enseignement et de recherche français ou étrangers, des laboratoires publics ou privés. 


\title{
L'habitat épimagdalénien des bruyères: essai d'analyse et d'interprétation
} des structures

\author{
par Bruno Bosselin
}

\section{I - AVANT-PROPOS}

Longtemps, la recherche archéologique a été axée vers la fouille de gisements stratifiés sous roche. Le but essentiel était de définir les civilisations, leur évolution et leur chronologie relative au détriment d'une étude paléthnographique approfondie.

Toutefois, les méthodes modernes ont renversé cette tendance : actuellement, les chercheurs considèrent les sites comme de véritables témoins de l'occupation humaine. L'approche des préhistoriques s'effectue par une analyse de leur mode de vie. D'illustres expériences ont ainsi apporté de fructueux résultats ; A. Leroi-Gourhan à Pincevent et $\mathrm{Y}$. Taborin à Etiolles entre autres (A. Leroi-Gourhan, 1972 et Y. Taborin, 1979). La représentativité reste cependant primordiale pour satisfaire des hypothèses sérieuses.

Ces nouvelles orientations se sont également révélées en Haute-Normandie lors de la prospection de vastes habitats de plein-air: Jean-Pierre Watté à Montivilliers et Gérard Fosse à Epouville (J.-P. Watté, 1975 et G. Fosse, 1978). Néanmoins, la répartition des sites paléolithiques dans cette région montre une densité de population importante à proximité des cours d'eau. La rareté et la pauvreté des gisements nous amène à nous demander s'il n'existe pas une lacune due à une zone d'intérêt des chercheurs plus faible. Enfin, la majorité des séries proviennent de ramassages de surface et ne reposent généralement pas sur des bases stratigraphiques solides (G. Fosse, 1978).

Le but de cette fouille était de déterminer la présence au sein d'un limon comprimé d'un groupe paléolithique supérieur et d'étudier l'organisation de l'habitat. Il semblait également que la plupart des découvertes régionales dataient d'un Paléolithique très primitif ou très final, imprégné d'influences nordiques mais encore sans traditions mésolithiques, rares : un seul gisement important, Vieilles et quelques microlithes dont l'attribution chronologique reste encore incertaine (J.-P. Watté, 1970). Une tentative de corrélation pouvait donc être intéressante (1).

\section{II - SITUATION TOPOGRAPHIQUE}

Le gisement de St-Pierre-du-Bosguérard se situe en rebord d'un petit plateau dominant celui du Neubourg d'une trentaine de mètres. Il est formé de limons quaternaires reposant sur une nappe d'argile à silex qui affleure sur les versants. Ceux-ci sont fortement marqués vers le nord et semblent avoir été aplanis au sud. Signalons un anticlinal orienté WNW-ESE qui prend en écharpe la vallée de l'Oison entre St-Amand-des-Hautes-Terres et St-Didier-desBois et passe à environ 500 mètres au nord du gisement (fig. 1). A ce niveau, le Cénomanien se trouve surélevé et forme une côte entre le Haut-du-Fec et Houlbec (2). Des affleurements de craie se rencontrent au nord sur 6 à $7 \mathrm{~km}$. Ils sont jalonnés, à leur base, par une ligne de sources.

(1) Un nouveau gisement mésolithique a été découvert en 1980 et est fouillé à Acquigny par G. Fosse et M.-A. Locard (renseignement oral de M. Fosse).

(2) D'après la notice explicative de la carte géologique au 1/50 000 de la région d'Elbeuf (B.R.G.M. 1972).

Coordonnées Lambert (Feuille Elbeuf 3-4 au 1/25 000) : $X=496.5$; $\mathrm{Y}=173.1 ; \mathrm{Z}=177$. 


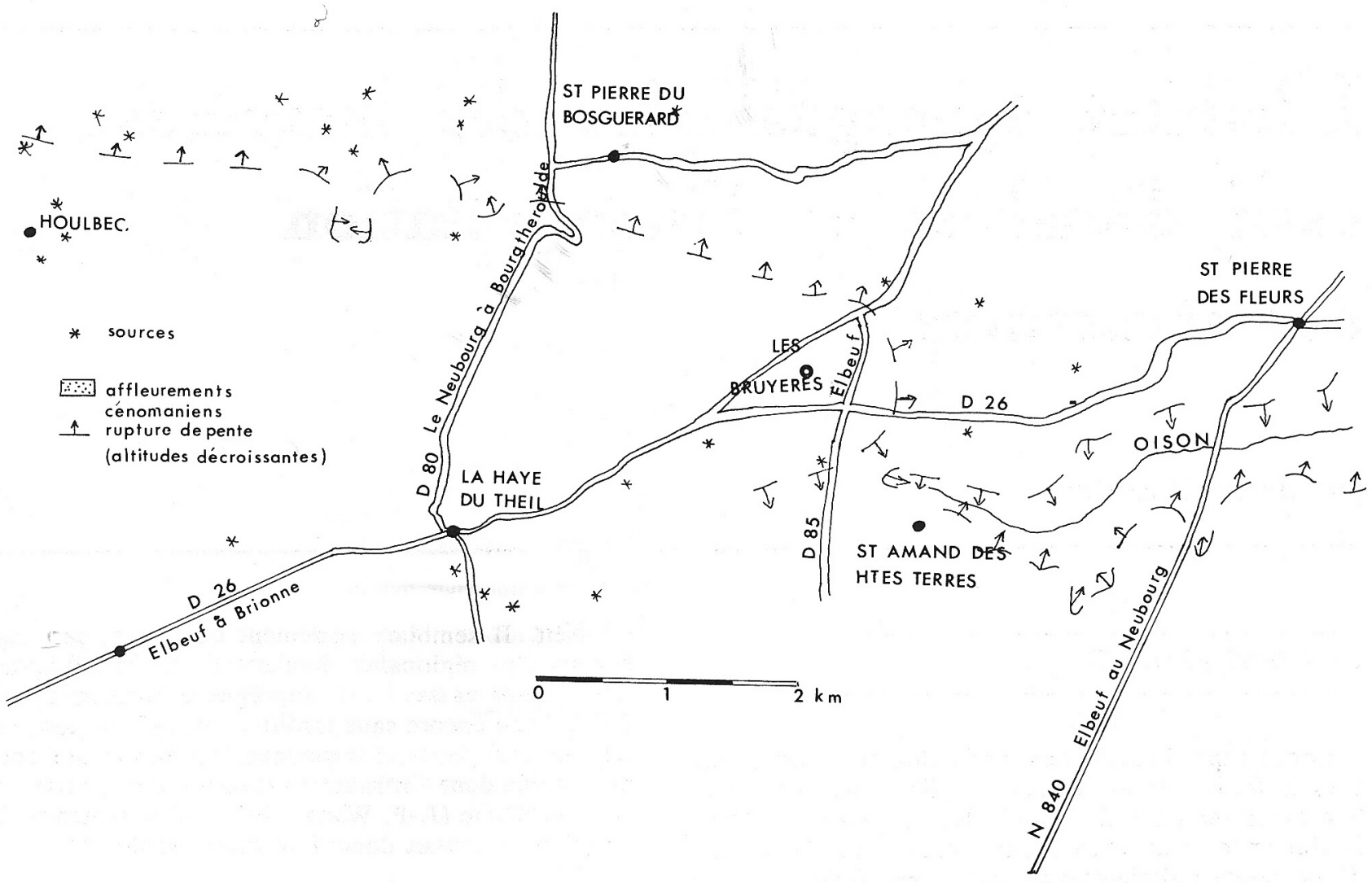

Fig. 1 - Situation du gisement des Bruyères à Saint-Pierre-du-Bosguérard (Eure).

\section{III - GÉOMORPHOLOGIE}

Le plateau du Neubourg, situé entre 130 et 160 mètres d'altitude, domine les vallées de la Risle à l'ouest, de l'Iton au sud et de l'Oison au nord-est. Le sous-sol est formé d'étages crétacés fortement décalcifiés. La couverture de limons quaternaires repose sur une nappe d'argile à silex tertiaire. A proximité des vallées, le limon s'est accumulé et quelques briquetteries ont pris naissance (St-Pierre-les-Elbeuf, Evreux, Bonneville, Appetot). Malgré tout, les découvertes relatives au Paléolithique restent encore rares (L. Coutil, 1893 ; A. Dubus, 1913, 1914, 1915 ; J.-P. Lautridou et G. Verron, 1970 ; B. Bosselin, 1978 [1981]).

\section{Stratigraphie}

Jean-Pierre Lautridou, chargé de recherches au Centre National de la Recherche Scientifique, du Centre de Géomorphologie de Caen, s'est rendu sur les lieux afin d'examiner les coupes disponibles. Il propose l'interprétation suivante de la stratigraphie observée (fig. 2) :

- Couche 1: Terre végétale grise formée par pédogénèse Elle contient quelques artéfacts en position secondaire.

- Couche 2: Limon orangé ; altération superficielle de la couche sous-jacente. Elle a livré de nombreux silex paléolithiques à sa base.

- Couche 3: Horizon majeur de la coupe. Il s'agit d'un limon brun hydromorphe très marbré. Il daterait de la fin du cycle würmien.

- Couche 4: Cailloutis de solifluxion contenant le Moustérien. Il serait vraisemblablement contemporain de l'interstade Würm $1 / 2$.

- Couche 5: Ancien limon argileux très altéré.

Le Paléolithique Supérieur se rencontre essentiellement à la base de la couche 2 et au sommet de la couche 3. Par sa position stratigraphique même, il semble correspondre à un faciès tardif. L'industrie trouvée in situ se compose de lames régulières, de nombreux éclats de débitage et de quelques outils de bonnes factures : deux lamelles à dos, deux pointes « aziliennes », un grattoir, un burin, un perçoir et une lame bitronquée. Des structures d'habitat ont été décelées au sommet de la couche 3 . 


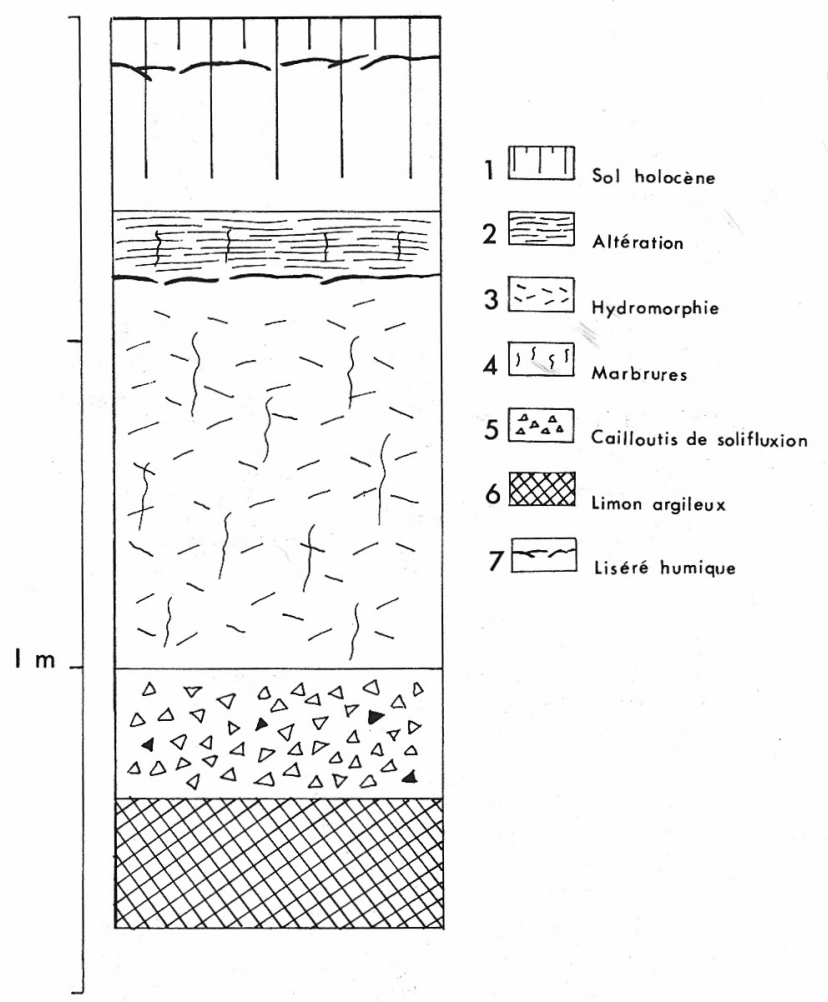

Fig. 2 - Coupe synthétique du gisement de Saint-Pierre-duBosguérard

\section{IV - DÉBITAGE}

\section{Décompte :}

$\begin{array}{lr}\text { Éclats } & 149 \\ \text { Lames } & 71 \\ \text { Cassons } & 142 \\ \text { Nucléus } & 21 \\ \text { Outils } & 35 \\ & -418\end{array}$

La matière première utilisée est un silex gris-noir, rarement blond, très doux au toucher. Une « pointe azilienne » est façonnée dans une roche marron qui ne semble pas originaire de la région. La patine est généralement blanche ou bleutée mais quelques pièces présentent une coloration ocre ou jaune.

La forte proportion de débris montre que le silex était de médiocre qualité et se brisait aisément à la percussion. Toutefois, les lames sont nombreuses et bien venues (ILam : 30,21\%).

L'étude des talons révèle une forte proportion de types dièdres et facettés (IF : 19,3\%).

Les nucléi sont principalement prismatiques et pyramidaux.
$\mathrm{V}-O U T I L L A G E$

L'étude typologique a été menée selon la méthode de Mme de Sonneville-Bordes (D. Sonneville-Bordes et J. Perrot, 1954, 1955, 1956). Les différents pourcentages ne sont donnés qu'à titre indicatif car il est difficile de tenter une approche statistique à partir d'un si faible échantillon.

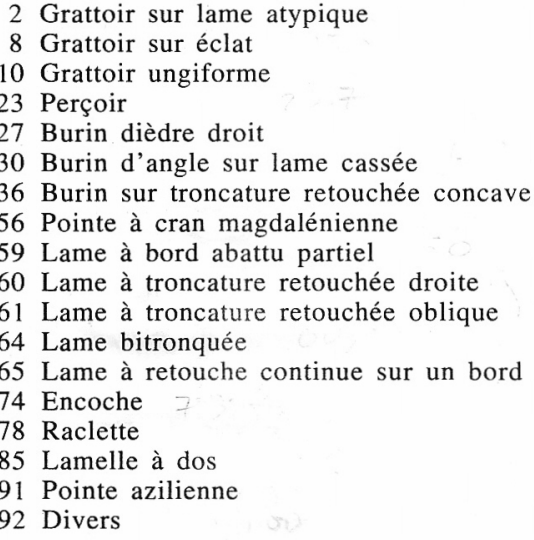

\section{Les Burins :}

Ils sont moyennement représentés (IB : 14,3\%). Les exemplaires dièdres (fig. $3, \mathrm{n}^{0} 1$ ) dépassent les types sur troncature (IBd : $11,43 \%$ et IBT : $2,86 \%$ ).

Ces outils sont généralement façonnés sur éclats courts, parfois massifs. Ils possèdent rarement deux pans ; l'autre est formé soit par une cassure, soit par un méplat naturel. Le biseau est toujours limité (fig. 3, $\mathrm{n}^{\circ}$ 6-17).

La seule troncature est concave et proche de l'encoche (fig. $3, \mathrm{n}^{\mathrm{o}} 15$ ).

\section{Les Grattoirs :}

Un peu plus communs que les burins (IG : $17,14 \%$ ), ils caractérisent parfaitement l'industrie de St-Pierre-du-Bosguérard. Des éclats courts, des lames cassées constituent l'essentiel des supports. Les types laminaires sont exceptionnels (fig. $3, \mathrm{n}^{\mathbf{0}}$ 10). Parmi les grattoirs courts, aucun ne possède vraiment le type azilien ; certains s'en rapprochent du point de vue morphologique (fig. $3, \mathrm{n}^{\mathrm{os}} 11$ à 13).

La retouche affecte rarement le bord latéral.

De petite taille, ils ont tous un front plat, convexe et régulier. Il n'y a pas de grattoir ogival ou à museau. L'un d'eux, sur éclat retouché, tend vers le type circulaire (fig. $3, n^{0} 13$ ). 

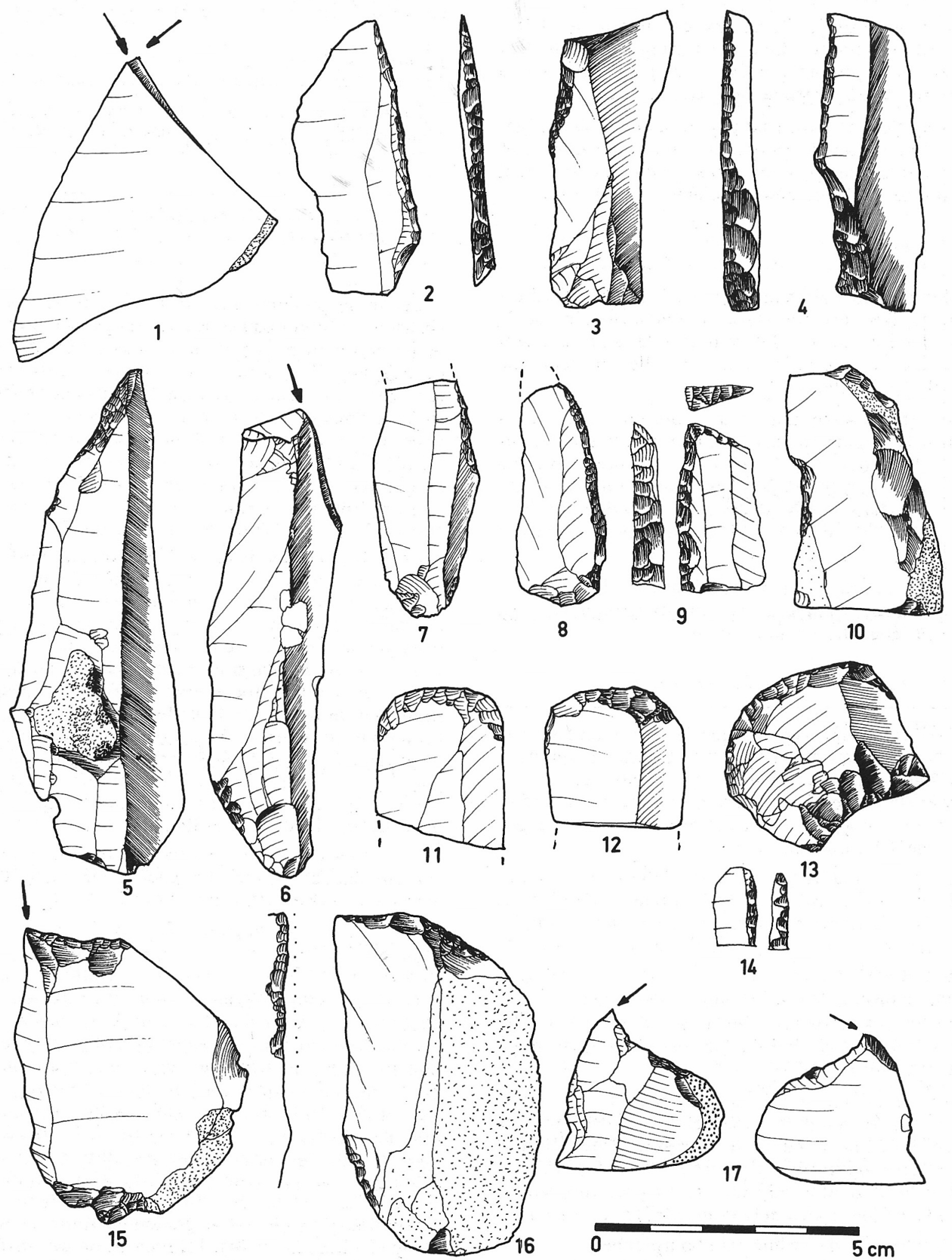

Fig. 3 - Industrie lithique du Paléolithiqua Supérieur. 
Les Perçoirs :

La proportion de perçoirs est importante (IP : $5,71 \%)$. Ce pourcentage nous rapproche fortement des cultures post-magdaléniennes plutôt que de la tradition magdalénienne proprement dite.

Il s'agit d'une part d'un perçoir à pointe fine dégagée par une retouche latérale semi-abrupte (fig. $3, \mathrm{n}^{\circ}$ 5). L'autre, plus grossier, nous évoque les becs dont l'extrémité est légèrement déjetée vers la droite.

\section{Les Troncatures :}

Elles sont très abondantes (Itr : $11^{1}, 43 \%$ ). Ceci est fréquent dans les civilisations septentrionales de la fin du Paléolithique. Malgré tout, elles sont rarement de bonne facture : deux d'entre elles portent une retouche partielle.

Par contre, une lame large présente des traces d'utilisation dans un secteur adjacent à la pointe, sur la face inférieure (fig. $3, \mathrm{n}^{0} 16$ ). Ce genre de pièce a déjà été rencontré par $\mathrm{M}$. Brézillon à Pincevent $(\mathrm{A}$. Leroi-Gourhan et al., 1966 ; B. Schmider, 1979). Elles ont probablement été utilisées pour le travail d'une matière dure.

Enfin, un fragment microlithique de lame $(22 \times$ $14 \times 3 \mathrm{~mm}$ ), tronqué à ses deux extrémités pourrait être une ébauche de microlithe.

\section{Les Pièces à dos et les Pièces à Cran:}

C'est le groupe typologique le plus intéressant et le plus important car il permet une localisation chronologique quasi-certaine. Cette industrie, par le nombre de pièces à dos et à cran,s'intègre vraisemblablement aux cultures à Federmesser du Tardiglaciaire de 1'Europe du Nord-Ouest.

Les pointes à dos sont assimilables aux pointes aziliennes du Sud-Ouest de la France. Cependant, elles s'en distinguent par un dos peu épais et faiblement incurvé. Leur longueur est toujours restreinte (fig. $3, \mathrm{n}^{0} 8$ ).

Deux lames cassées portent une retouche abrupte partielle très soignée. Ce sont sans doute là des pièces brisées lors de leur fabrication puis abandonnées (fig. $3, \mathrm{n}^{\text {os }} 3-7$ ).

Le pourcentage de pièces à dos et à cran est important si on le compare avec celui des gisements du Bassin Parisien et même du Nord-Ouest de l'Europe jamais en proportion si forte ( $\mathrm{ICr}: 5,71 \%$ ). Ils sont associées à un dos adjacent rectiligne, très soigné (fig. 3, nos 2-4) (B. Schmider, 1971).

Il n'y a pas de pointe hambourgienne.

Enfin, deux pointes aziliennes sont tronquées (fig. $3, n^{0} 9$ ). Les lamelles à dos (fig. $3, n^{0} 14$ ) très abondantes dans le Magdalénien classique, semblent en défaut dans la série de St-Pierre-du-Bosguérard.

\section{L'outillage divers :}

On remarque l'abondance des encoches, la présence d'une raclette et la fréquence des lames retouchées.

VI - ST-PIERRE-DU-BOSGUÉRARD ET LE MAGDALÉNIEN FINAL DE HAUTE-NORMANDIE

La faible prédominance des grattoirs sur les burins, des grattoirs courts sur les longs et la présence de pointes aziliennes placent l'industrie de St-Pierredu-Bosguérard parmi les cultures post-magdaléniennes. Cependant, le caractère exceptionnel des fossiles directeurs de l'Azilien nous amène à considérer des civilisations installées au Nord-Ouest de l'Europe au Tardiglaciaire final. Les vestiges du $\mathrm{Pa}-$ léolithique Supérieur, rares en Haute-Normandie, se situent principalement lors de l'amélioration climatique de la fin du Würm (G. Fosse, 1978).

En 1910, Gadeau de Kerville et Poulain ont fouillé avec succès un abri sous roche près de Bonnières (Gadeau de Kerville et Poulain, 1910). Le matériel recueilli : des lames, des lamelles mais seulement trois outils se trouvaient dans une couche argilocalcaire à $70 \mathrm{~cm}$ du sol. "Un foyer (a été trouvé) dans un creux de la roche vive. A côté de lui, étaient beaucoup de lames, de lamelles et d'éclats en petits tas autour du foyer ». Il est difficile de comparer ce matériel avec celui de St-Pierre-du-Bosguérard étant donnée la faiblesse du lot. L'attribution chronologique d'A.-G. Poulain au Magdalénien semble confirmée par la faune et l'outillage osseux.

A St-Pierre-d'Autils, Poulain a mis au jour une station magdalénienne. Là encore, le matériel est extrêmement rare (Poulain, 1902 à 1912).

A 600 mètres de la gare d'Évreux, F. Bordes et P. Fitte ont découvert une industrie épipaléolithique ( $\mathrm{F}$. Bordes et P. Fitte, 1951). Les grattoirs, surtout courts, dépassent nettement les burins rarement typiques. La proportion de troncatures et de pièces à dos est importante. Elles se rapprochent des pointes aziliennes : il n'y a aucun dos anguleux ou à cran. Les encoches et denticulés sont très nombreux. Des structures ont été décelées dans un limon postérieur au loess récent IV. Cela a permis à $F$. Bordes de classer cette industrie parmi les cultures épipaléolithiques bien qu'aucun microlithe n'ait été récolté. Il a ensuite modulé son affirmation en suggérant une attribution possible au Magdalénien final, comme à Gouy (F. Bordes, 1974). La répartition des différentes catégories est similaire; les encoches et pièces à dos sont plus fréquentes à Évreux tandis que le rap- 


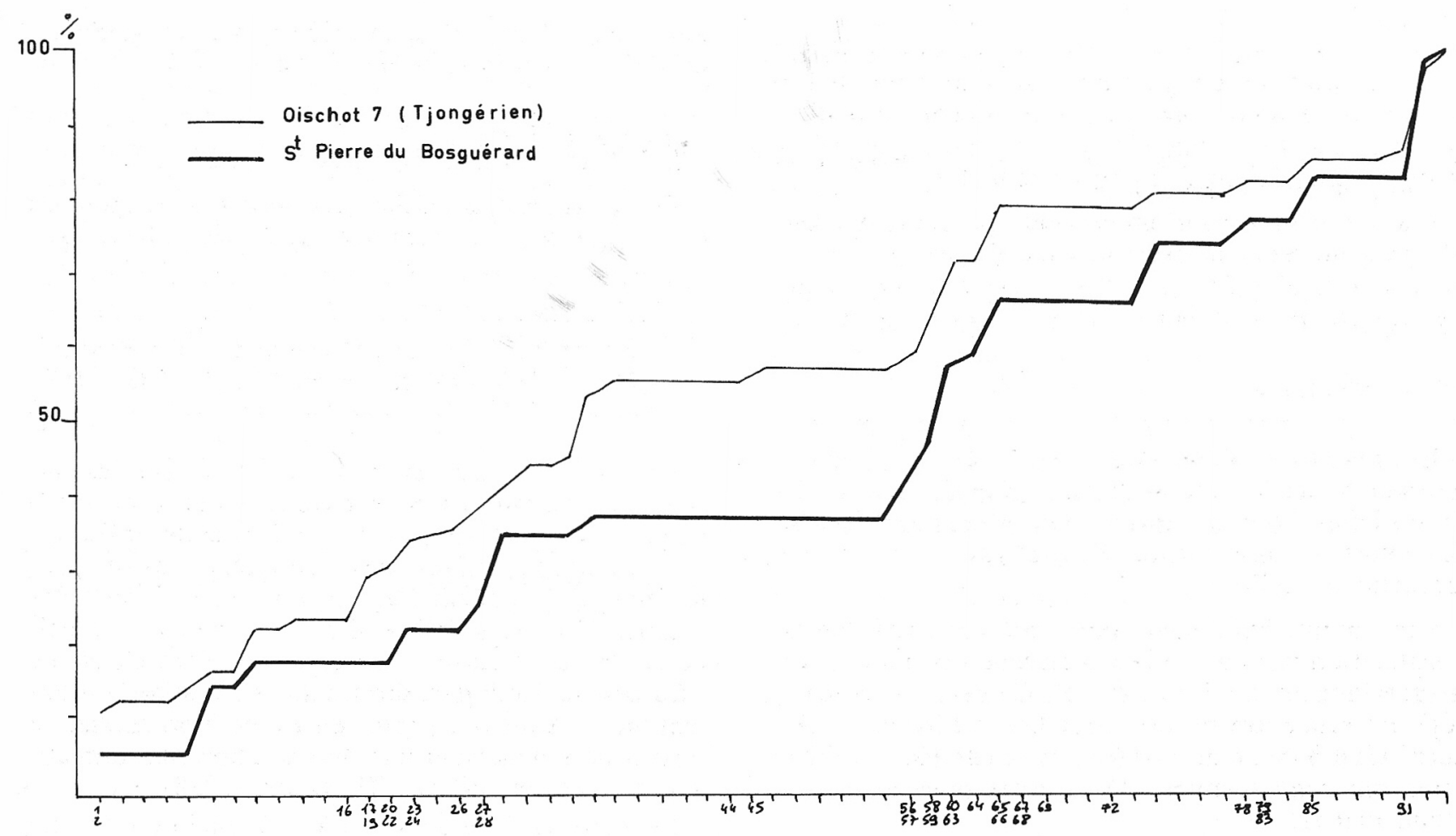

Fig. 4 - Comparaison avec le Tjongérien.

port G/B est le même. L'absence de cran place ce gisement dans la tradition périgourdine et l'éloigne des influences nordiques.

La grotte du Mont Criquet à Gouy, située dans la craie sénonienne à $10 \mathrm{~km}$ en aval de Rouen, est célèbre pour ses gravures. Le matériel lithique se compose de nombreuses lames, un nucléus et 16 outils. Les grattoirs, peu typiques, sont en nombre légèrement plus faible que les burins, surtout sur cassure. Les pointes aziliennes abondent ; on note un dos anguleux cassé formant peut-être initialement un cran. Les lames tronquées attestent d'une utilisation. Là encore, les encoches et denticulés sont nombreux. $\mathrm{Ce}$ gisement et St-Pierre-du-Bosguérard ont beaucoup de points communs bien que les burins soient plus fréquents que les grattoirs à Gouy. On retrouve les pièces à dos, l'existence de dos anguleux, la fréquence des lames tronquées et des burins sur cassure. D'après le style des gravures rupestres, il s'agirait d'un Paléolithique final, sans doute contemporain de notre gisement (F. Bordes, 1974).

Le gisement de Vattetot-sous-Beaumont, au lieudit La Gripperie, se trouve dans la même situation topographique ; rebord d'un plateau de limons quaternaires, présence d'une formation à silex sur les versants, épaisseur de limon de plus de 1,5 m. Les deux séries lithiques ont de multiples similarités; nombreux grattoirs, sur bout de lames cassées, burins sur cassure et pièces à dos. Bien que cette série soit limitée, son rattachement à un stade évolué du Paléolithique est plus que probable. « Il n'est d'ailleurs pas exclu que l'on puisse trouver un jour des éléments autorisant un rapprochement avec les faciès nordiques » (G. Fosse, 1978).

Les grands gisements de la fin de la glaciation de Würm sont assez rares en Haute-Normandie. Les séries lithiques, toujours limitées à une trentaine de pièces au maximum, permettent de déduire les caractères généraux de cette période de transition :

- Les grattoirs sont plus abondants que les burins. Ils ont subi une forte réduction de taille et ont pour support des éclats ou lames courtes. Les burins ne présentent fréquemment qu'un seul enlèvement; burin sur cassure ou sur pan naturel.

- Les lames à troncature apparaissent comme un groupe typologique stable et important (7 à $10 \%$ ).

- Les pièces à dos sont de loin les types d'outils les plus fréquents. Elles se trouvent sous de nombreuses formes : pointes aziliennes, lame à dos mais également pointe à dos anguleux ou à cran. On retrouve ici les influences venues du Nord de l'Europe. 


\section{VIII - COMPARAISON AVEC LE MAGDALÉNIEN FRANÇAIS (fig. 5)}

Dans le Bassin Parisien, A. Leroi-Gourhan a démontré une certaine variété dans les industries. Il a, entre autre, divisé le Magdalénien supérieur et final en deux groupes qui ont, semble-t-il, vécu à Pincevent.

- Le premier se caractérise par la faiblesse des lamelles à dos, l'importance des lames tronquées et des becs ; cela correspond à $1^{\prime}$ habitation $\mathrm{n}^{\mathbf{0}} 1$ de Pincevent (A. Leroi-Gourhan, 1966).

- L'autre, représenté par la section 36, donc un peu plus récent se rapproche plutôt du Magdalénien classique avec de nombreuses lamelles à dos, des burins bec-de-flûte et des micro-perçoirs, encore nombreux (A. Leroi-Gourhan, 1972).

St-Pierre-du-Bosguérard se rattacherait au premier groupe bien que certains caractères soient différents : les burins sont plus nombreux que les grattoirs et les zinken abondent. Toutefois, l'échantillon des pièces à cran nous suggère d'une part une filiation, d'autre part une implantation plus tardive. Ce gisement constituerait un faciès évolué de cette lignée.
En ce qui concerne le Magdalénien classique du Périgord, il n'existe pratiquement pas de points communs : les lamelles à dos et les burins sont pratiquement les seuls outils en forte proportion. Les grattoirs et les troncatures atteignent rarement plus de $10 \%$. Par contre, l'Azilien possède les mêmes paramètres ; les lamelles à dos ont presque disparu, les burins ont subi une réduction importante et se développent massivement les grattoirs et pointes aziliennes (D. Sonneville-Bordes, 1960). Les seuls gisements comparables se rattachent à un Azilien primitif, dont certains ne sont pas à l'abri d'influences nordiques, moins marquées que dans le Bassin Parisien (A. Chollet, 1979 ; J.-M. Le Tensorer et al., 1979).

\section{IX - ESSAI D'ANALYSE ET D'INTERPRÉTATION DES STRUCTURES (fig. 6)}

Sur la totalité de la surface fouillée, soit $21 \mathrm{~m}^{2}$, la couche archéologique est englobée au sein d'un limon brun hydromorphe de la fin du cycle würmien. $\mathrm{Ce}$ niveau est bien individualisé et homogène sur

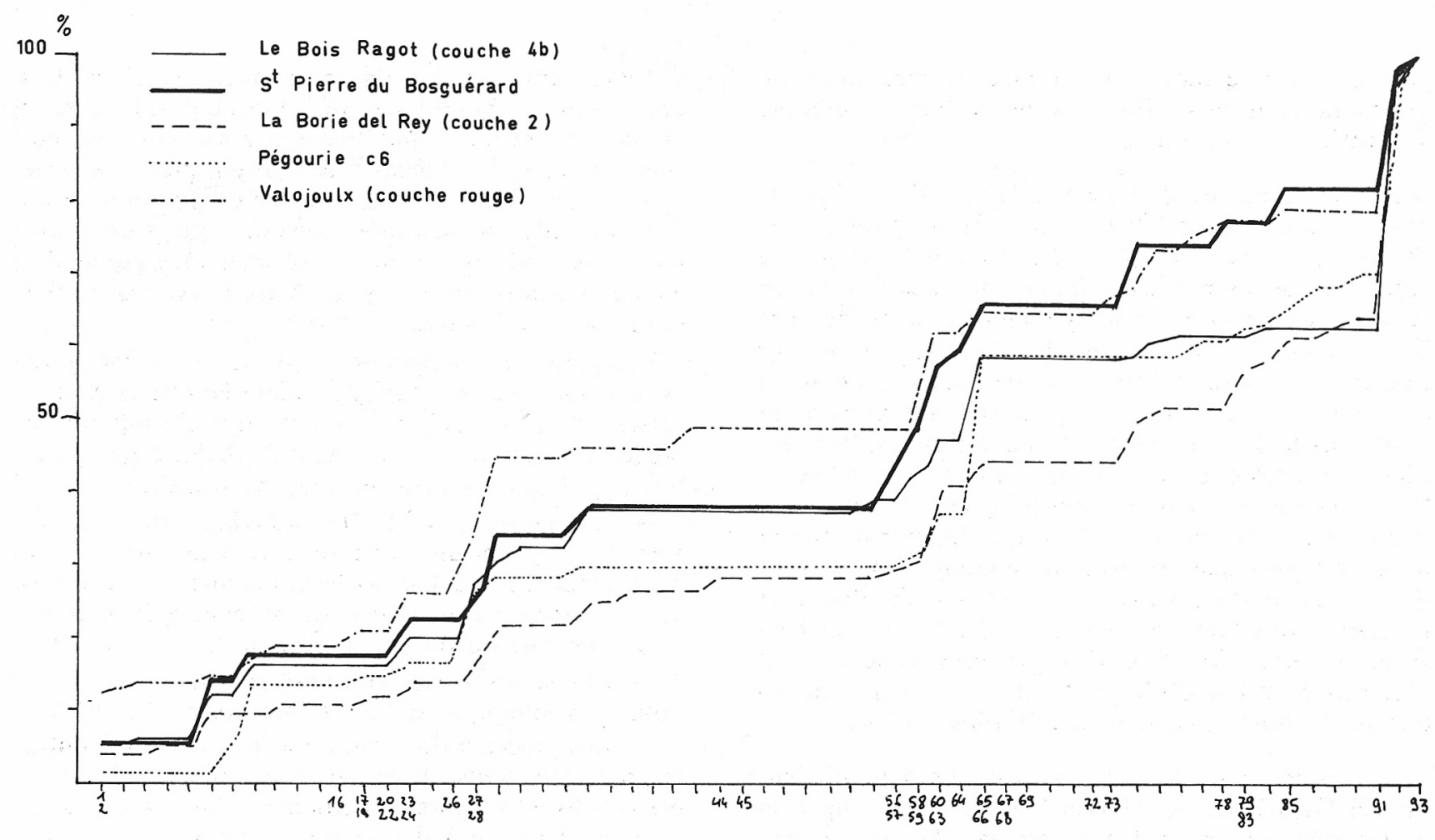

Fig. 5 - Comparaison avec le Magdalénien et l'Azilien français. 
près de $20 \mathrm{~cm}$. On remarque un léger lessivage dans la couche. Les éléments structurés se présentent sous quatre formes:

- Deux blocs très compacts constitués indifféremment de graviers et de pierres de $10 \mathrm{~cm}$ environ. Leur altitude est supérieure à celle des autres vestiges (Carrés K 12 et M 14).

- Des alignements plus ou moins compacts de grosses pierres très altérées ; beige en surface et ocre rouge en profondeur. Les pans de mur ainsi formés ont été parfois fortement perturbés par les racines. Il ne semble pas qu'il s'agisse de silex ou alors très corrodés par le milieu acide (Carrés K 12 - L 13 M 12 et $\mathrm{N}$ 12).

- De fortes concentrations de petits cailloux intimement mêlés à la matrice limoneuse et assurant une continuité avec les éléments décrits auparavant.

- Deux petits cercles de pierres semblent délimiter un espace dans lesquels ont été remarquées quelques traces cendreuses. On ne peut pas parler de foyer car cela supposerait une organisation complexe et une rubéfaction importante qui n'existe pas. Il n'est pas étonnant de ne pas retrouver cette rubéfaction qui s'altère très vite dans le limon (Communication orale de G. Fosse, A. Michel et J.-P. Watté à la Société Normande d'Etudes Préhistoriques et Historiques le 14 décembre 1980 ).

La plupart des éléments gịsaient bien à plat mis à part deux petites structures circulaires décrites auparavant et quelques-uns manifestement déplacés ultérieurement à l'abandon du gisement par les préhistoriques. La taille de ceux-ci, la présence d'un amas de débitage non perturbé interdisent d'envisager une mise èn place de l'ensemble par solifluxion. On doit admettre l'existence d'un campement paléolithique à cet endroit.

La conservation de la structure semble avoir été meilleure au Nord et au Nord-Est, tandis que de graves endommagements ont été décelés au Sud et à l'Ouest. La majorité des silex taillés, outre l'amas de

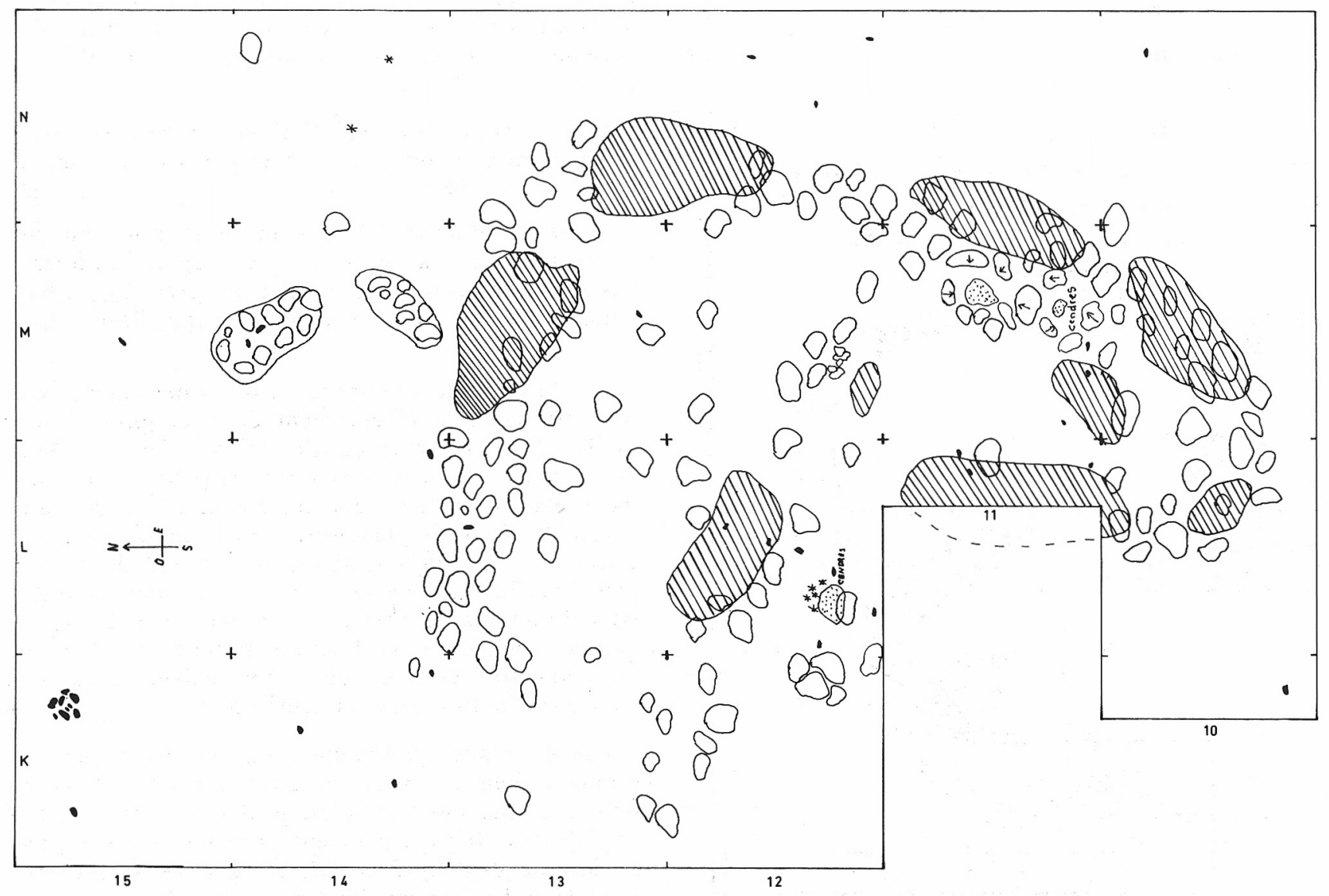

Fig. 6 - Répartition des vestiges : les pierres sont figurées en blanc, les silex en noir. Les hachures représentent les zones de cailloutis. 
débitage, gisaient à l'extérieur bien à plat, rares étaient ceux présentant un pendage important (3). Enfin, une lame à dos cortical était figée entre deux rognons dans une partie du mur (carré L 13).

\section{1) Essai d'interprétation}

La forme générale qui se dégage est celle d'un ovale proche du fer à cheval; un côté concave. Il semble s'agir d'une structure assez réduite : environ $2,5 \times 3,5 \mathrm{~m}$. Il n'est pas possible de préciser davantage étant donné l'état de conservation.

Les deux blocs plus compacts situés au Nord et à l'Ouest de l'habitat correspondent à des pierres de calage. Ils n'ont pas subi de perturbations.

Les deux cercles à l'intérieur de la structure pourraient être les témoins de foyer : la prudence en ce qui les concerne est recommandée car ils sont assez

(3) Sur 36 artefacts recueillis en 1980, 33 étaient à plat, 2 obliques et un seul était vertical.

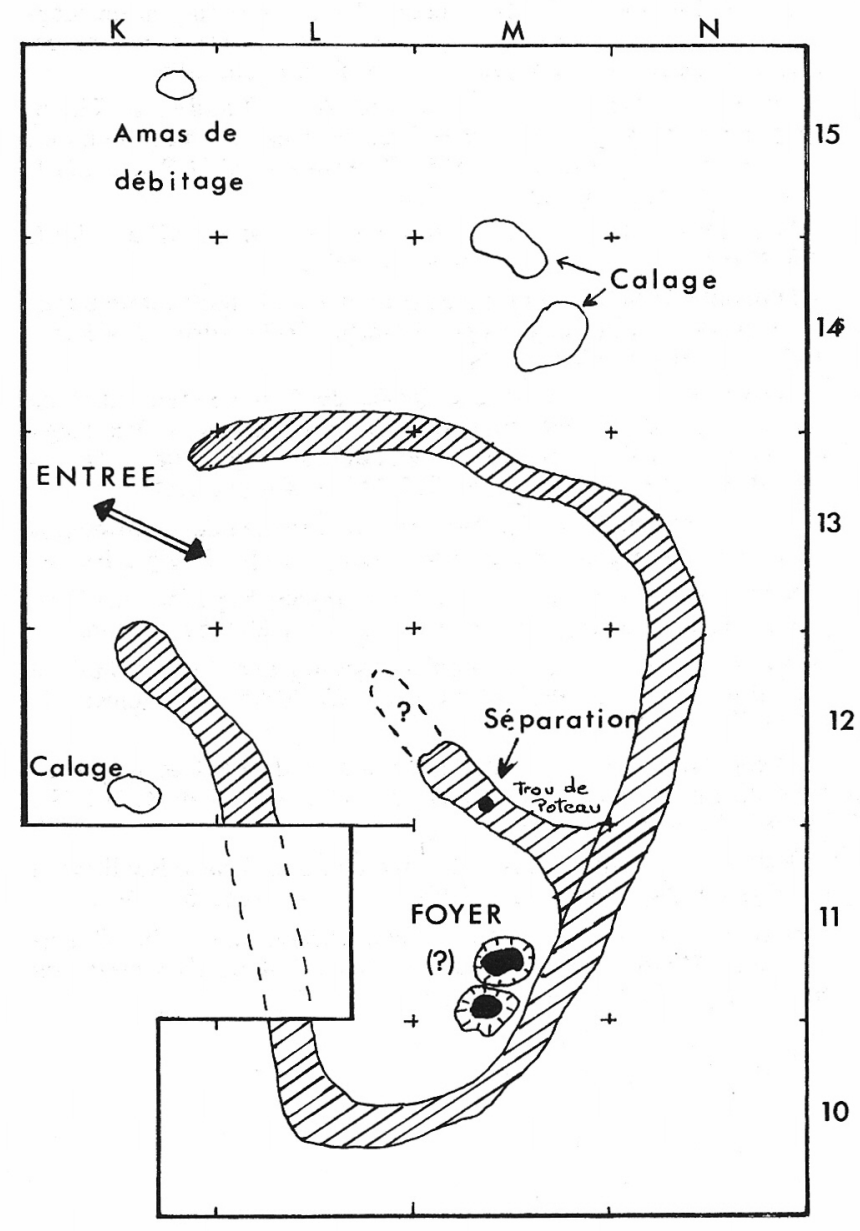

Fig. 7 - Interprétation des structures peu typiques. Les cendres recueillies sont très lessivées.

Un alignement intérieur semble délimiter une séparation hypothétique. On ne peut évidemment pas se fier aux différences de concentration des vestiges ; ceux-ci étant trop peu nombreux.

L'entrée se trouve dans le carré K 13.

Ce type de construction se retrouve, pendant la même période, en Allemagne. Ce sont les témoins de l'occupation des groupes à Federmesser : Hambourgien, Tjongérien et Ahrensbourgien.

\section{2) Écologie humaine}

La rareté des gisements, la pauvreté des séries lithiques conduisent à une étude écologique superficielle. Cependant, on peut avancer que les différents sites de cette époque ont certainement eu des rapports entre eux. Il n'est d'ailleurs pas exclu qu'il s'agisse de diverses manifestations d'un même groupe. L'avenir, c'est-à-dire la découverte de gisements similaires, nous réserve sans doute de bonnes surprises. Peut-être existe-t-il quelque part un lieu de rassemblement digne des grands gisements du Bassin Parisien.

L'installation des paléolithiques à St-Pierre-duBosguérard s'explique facilement par divers facteurs (A. d'Anna, 1980) :

- Du point de vue climatique, ils se trouvaient en pleine phase de transgression et de dégel. Les terres basses limoneuses devaient être gorgées d'eau. La situation en rebord de plateau les isolait de l'humidité.

- Du point de vue pratique, ils avaient une position privilégiée; affleurement de silex proches et faciles d'accès, présence d'un cours d'eau à $1 \mathrm{~km}$ environ et surveillance des alentours pour les mouvements du gibier. Il est évident que ce territoire était favorable à une implantation d'un groupe nomade. Enfin, la forêt, partout dominante, offrait de multiples possibilités : chasse, cueillette et ramassage de bois. L'étude du contexte montre que les préhistoriques avaient un rayon d'action limité à une dizaine de kilomètres et donc qu'il doit exister dans les environs d'autres habitats semblables.

Après l'étude typologique, on remarque que ce groupe n'était pas isolé : en effet, les outils de type nordique nous montrent bien qu'il y a eu des échanges d'idées. Il est également probable que certains paléolithiques de St-Pierre-du-Bosguérard soient responsables d'autres manifestations dans les environs, peut-être d'autres séries lithiques. 


\section{$\mathrm{X}-$ CONCLUSION - DATATION}

La station de St-Pierre-du-Bosguérard a livré une industrie de la fin du Paléolithique. D'après la répartition de l'outillage lithique, elle possède certains caractères du Magdalénien du Bassin Parisien et surtout des groupes à Federmesser d'Allemagne. Ces deux communautés déterminent ainsi un ensemble original situé à mi-chemin entre le Périgord et la zone nordique. L'attribution chronologique encore incertaine, pourrait se placer pendant 1'oscillation d'Allerid ou au début de Dryas III (10 000 à $9000 \mathrm{BC})$.

S'il est possible de reconstituer l'habitat de StPierre-du-Bosguérard, quelques détails restent encore inexpliqués :

- L'absence de véritable foyer et l'hypothèse d'un débitage sur place, vraisemblable, orientent les recherches vers la découverte de nouvelles structures.

Sans doute existe-t-il d'autres témoins de l'occupation de ces hommes (4).

Bruno BosSELIN, Résidence du Soleil, Rue de Vénus, St-Pierre-du-Bosguérard, 27370 Amfreville-la-Campagne.

\section{BIBLIOGRAPHIE}

D'AnNa A. et Mills N.-T.-W. - L'occupation néolithique du bassin de Trets. Bull. Arch. Provence, à paraître

Bohmers A. - Statistiques et graphiques dans l'étude des industries lithiques préhistoriques. Considérations générales sur le Hambourgien, Tjongérien, Magdalénien et Azilien. Palaeohistoria, vol. 8, 1961, pp. 15-37, 7 fig., 1 pl.

BORDES F. - Une industrie épipaléolithique à Évreux. Bull. Soc. Préhist. Franç., tome 48, 1951, pp. 147-154, 3 fig.

Bordes F. - Les limons quaternaires du bassin de la Seine. Stratigraphie et archéologie préhistorique. Archives I.P.H., mémoire 26, 1954, 473 p., 175 fig., 34 tabl., 1 carte hors-texte.

Bordes F. - L'industrie de la grotte ornée de Gouy. Bull. Soc. Préhist. Franç., tome $71, \mathrm{n}^{0} 4,1974$, pp. 115-118, 2 fig.

(4) Remerciements. - Je tiens à remercier Mme H. CAMPS-FABRER et M. A. d'ANNA pour la réflexion qu'ils ont fait naître en moi ; M. J.-P. WATTÉ pour son aide et ses encouragements, M. L. PERSONNAT pour la compréhension dont il a fait preuve ainsi que tous les fouilleurs. Qu'ils trouvent tous ici un hommage sincère.
Bosselin B - Eléments moustériens de Saint-Pierre-duBosguérard. Bull. Soc. Norm. Études Préhist. et Hist., tome 44 , $\mathrm{n}^{\circ}$ 3, 1978 (1981), pp. 2-12, 4 fig., 1 tabl.

Chollet A. et al. - La grotte du Bois Ragot à Gouex (Vienne) : industrie, géologie et paléontologie. La fin des Temps Glaciaires, Colloque $\mathrm{n}^{\mathrm{o}}$ 271, C.N.R.S., 1979, tome I, pp. 365 378, 8 fig., 3 tabl.

Collcutt S.-N. - Note sur le «L.U.P.»: Creswellien, Cheddarien de la Grande-Bretagne, ibid., tome 2, pp. 783-789, 1 fig., 1 tabl.

Coutil L. - Résumé des recherches préhistoriques en HauteNormandie : époque paléolithique. Bull. Soc. Norm. Ét. Préhist. et Hist., tome I, 1894, pp. 34-140, fig. et coupes.

Dubus A. - Carte pré- et protohistorique de la SeineInférieure. Soc. Géol. Norm., tome 33, 1913-1914-1915, pp I-III.

Fosse G. - Le Paléolithique supérieur de Haute-Normandie. Soc. Norm. Ét. Préhist. et Hist., tome 44, no 4, 1978, pp. 23-33, 4 fig.

Gadeau de Kerville H. et Poulain A.-G. - Résultats des fouilles effectuées dans un abri sous roche près de Bonnières, ibid., tome 18, 1910.

Lautridou J.-R. et Verron G. - Paléosols et loess de StPierre-les-Elbeuf. A.F.E.Q., no 3, 1970, pp. 145-165.

LE TENSORER J.-M. et SÉRONIE-VIVIEn M.-R. - Données récentes sur l'Azilien du Lot : la grotte de Pégourie à Caniac-duCausse. La fin des Temps glaciaires, Colloque 271 du C.N.R.S., 1979 , tome I, pp. 471-482, 15 fig., 1 tabl.

LEROI-GOURHAN A. et BREZILlON M.-N. - L'habitation magdalénienne $\mathrm{n}^{\circ} 1$ de Pincevent près Montereau (Seine-et-Marne). Gallia Préhistoire, tome 9, no 2, 1966, pp. 263-385.

Leroi-Gourhan A. et BreziLlon M.-N. - Fouilles de Pincevent. Essai d'analyse ethnographique d'un habitat magdalénien la section 36. $7^{\mathrm{e}}$ supp. à Gallia Préhistoire, C.N.R.S , 1972 331 p., 199 fig., 4 annexes, Plans.

Poulain A.-G. - Les fouilles de Mestreville. Bull. S.N.E.P.H., 1902 à 1912, Divers articles.

SChmider B. - Les industries lithiques du Paléolithique Supérieur en Ile de France. $6^{\mathrm{e}}$ supp. à Gallia Préhistoire, C.N.R.S., 1971,218 p., 109 fig., 9 tabl.

SCHMIDER B. - Un noveau faciès du Magdalénien final du Bassin Parisien : l'industrie du Pré des Forges à Marsangy (Yonne). La fin des temps glaciaires, Colloque 271 du C.N.R.S., 1979, tome 2, pp. 763-771, 4 fig., 1 tabl.

SCHWABEDISSEN H. - Die Federmesser Gruppen des nordwesteuropaischen Flachlandes. Neumûnster, 1954, 104 p., 23 fig., 106 pl.

Sonneville-Bordes D. - Le Paléolithique Supérieur en Périgord. Imp. Delmas, Bordeaux, 1960, 558 p., 294 fig., 2 tomes.

SONNEVILlE-Bordes D. - Lexique typologique du Paléolithique supérieur. Bull. Soc. Préhist. Franç., 1954-1955-1956, tomes 5152-53

TABORIN Y. - Les habitats paléolithiques du bord de la Seine : Etiolles. La fin des temps glaciaires, Colloque 271 du C.N.R.S., tome 2, 1979, pp. 773-781, 7 fig.

WATtÉ J.-P. - Microburins et « microlithes » dans le Néolithique du Pays de Caux. Bull. Soc. Préhist. Franç, tome 67, 1970.

WATtÉ J.-P. - L'habitat « Seine-Oise-Marne» du GrandEpaville à Montivilliers : premiers résultats. Annales du Muséum du Havre, supp. 2, Juin 1975, 11 p. 